\title{
AN UNUSUAL FORM OF WIDESPREAD VASCULAR DISEASE OF THE BRAIN IN A YOUTH
}

\author{
BY \\ C. S. TREIP and R. J. PORTER \\ From the Central Middlesex Hospital, London
}

The protean manifestations of vascular disease of the brain are well known. The following case seems worthy of report because of the unusual clinical picture and its consequent diagnostic difficulties, and the diagnostic problems raised by the lesions found at necropsy.

\section{Case Report}

The patient was a young man aged 19 years at the time of death. There was no relevant family history. There was one sibling, a sister aged 21 , who was quite healthy. The patient was a non-smoker and a teetotaler. $\mathrm{He}$ had been a perfectly normal boy of clearly high intelligence and had had no significant physical disease until the onset of his present illness.

At the age of 14 he passed the School Certificate examination with five credits and in the same year won a scholarship to a public school. He did well there but in July, 1950, at the age of 18 , he failed the Higher Certificate examination quite unexpectedly in one subject. In September, 1950, he joined the Army and at first seemed to do well. In February, 1951, while on leave, his parents thought he was a little forgetful and noticed that he was "fidgety". In March, 1951, he was posted to Germany and soon afterwards it was noticed that his behaviour was unusual. He seemed forgetful and, on one occasion, was put on a charge for losing his rifle.

In July, 1951, his letters home, which had previously been regular, suddenly ceased, and soon afterwards his parents were notified that he had been admitted to hospital with a nervous breakdown. He was invalided home to Netley Military Hospital, where a diagnosis of early schizophrenia was made and he was given E.C.T. His parents were quite shocked when they visited him there, and said he looked like someone from Belsen, thin and shaky. He was discharged and returned home in October, 1951. By this time his physical condition had greatly improved; he had put on weight and looked well nourished. His memory and powers of concentration, however, were grossly defective and he was unable to interest himself in anything. He was unsteady in his gait and very clumsy with his hands. He also had a tendency to bump into things, and his father suspected that his eyesight was failing. On two occasions he had been incontinent of urine in the night and, when rebuked about it, had laughed. It was also noticed that he was excessively drowsy and, the day before admission to hospital, he had complained of pins and needles down the left side of the body. There had been no complaint of headache and no vomiting.

He was referred for a further psychiatric opinion to Dr. Haldane at the West Middlesex Hospital, who found him to be obese, fatuous, and incoordinate, with slurred speech and ataxic gait. It was considered that he was suffering from an organic dementia. He was admitted to the Central Middlesex Hospital on November 2, 1951, for further investigation.

On examination he was very slow in his thoughts, mildly euphoric, had a very poor memory and was unable to give any satisfactory history. He admitted to̊ being ill for only two months. He remembered that he had got into trouble in the Army for forgetfulness and had been punished, and that he had been boarded outo as a psychiatric case. He denied headache and seemeos to be very indifferent to his present disability. There was no dysphasia but speech was slurred. The left eye was completely blind and the disc showed advanced primary optic atrophy. The right optic disc appeared normal but there was a complete temporal hemianopia on this side. The pupils were equal, the left was fixed to direct light but reacted briskly consensually. The right pupil reacted well to direct light. There was no nystagmus, and the other cranial nerves were normal. The left arm was slightly spastic and weakness, with marked incoordination and intention tremor on this side. The tendon reflexes were all brisk, perhaps a little brisker left than right, and the left plantar response was extensor. There was no objective sensory abnormality. The gait was grossly ataxic. There was clinical evidence of marked cardiovascular hypertrophy and the blood pressure was $190 / 130 \mathrm{~mm}$. Hg. There were no abnormal physical signs on examination of the other systems.

Investigations.-A radiograph of the chest confirmed gross left-sided cardiac enlargement. The urine contained a cloud of albumin, a few red cells, and occasional granular casts. Lumbar puncture revealed a clear fluid, pressure $120 \mathrm{~mm}$.; the protein was $80 \mathrm{mg}$. per $100 \mathrm{ml}$., the fluid otherwise being normal. Wassermann reactions of the C.S.F. and blood were negative. The blood urea level was $41 \mathrm{mg}$. per $100 \mathrm{ml}$. A blood count gave: haemoglobin $94 \%$, W.B.C. 8,000 (neurtophils $75 \%$, 
eosinophils $2 \%$, lymphocytes $20 \%$, monocytes $3 \%$ ). An intravenous pyelogram showed separate upper poles of both kidneys with a double ureter on both sides. The excretion from both kidneys was good. A screening test for phaeochromocytoma, using $20 \mathrm{mg}$. of methylpiperidyl benzodioxane, was negative. A straight radiograph of the skull was normal. An electroencephalogram showed a generalized abnormality with a very slight right-sided preponderance. Delta rhythm was dominant in all areas and of moderate voltage. Traces of lowvoltage alpha rhythm of 8 to 10 c.p.s. were present. The slow activity was of a rhythmical character and clean outline. The tracing was thought to be compatible with either a subtentorial tumour or a generalized degenerative lesion of both hemispheres.

Progress.-Soon after admission to hospital he became drowsy and unresponsive, and a posterior fossa tumour was considered as a possible diagnosis. On December 11, a ventriculogram was done by Mr. T. G. I. James. This showed the lateral and third ventricles filling well; they were not displaced. The third ventricle was somewhat dilated; the fourth ventricle and aqueduct were not filled. A considerable quantity of air was shown in the subdural space and was thought to indicate some degree of cortical atrophy. Three millilitres of " myodil" were injected in an attempt to fill the aqueduct and fourth ventricle but failed to do so. The posterior fossa was therefore explored. No tumour or obstruction was found

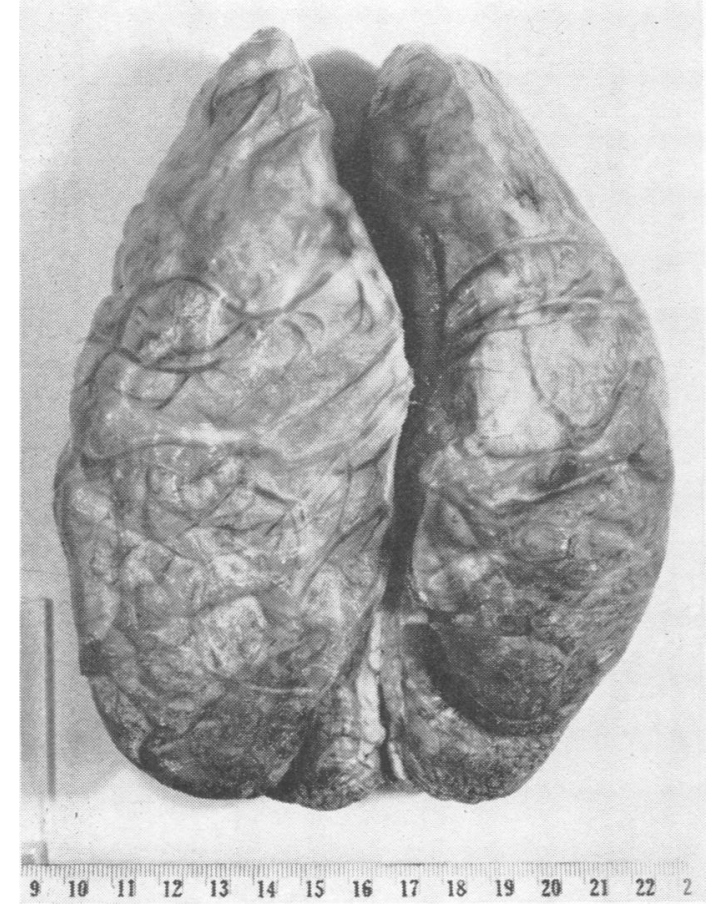

FIG. 1.-General view of the vertex, showing shrinkage of the frontal lobes and leptomeningeal thickening. and droplets of " myodil " were seen in a dilated fourth ventricle. The cerebellum appeared to be smaller than usual.

After the exploration he seemed to improve temporarily; he was able to sit up and speak, but 29 days after the operation he lapsed into coma, developed increasing neck rigidity and eventually decerebrate rigidity and opisthotonus. He died in this state on March 3, 1952, 82 days after operation and about 13 months after the onset of symptoms.

Necropsy.-Post-mortem examination was performed 36 hours after death by H.M. Coroner's pathologist at the Central Middlesex Hospital. The body was that of a very emaciated young adult man. Externally the brain weighed $1,000 \mathrm{~g}$. and was very shrunken, shrinkage being more marked in the cerebrum. The leptomeninges were greatly thickened over the frontal area (Fig. 1), and showed much staining by haemosiderin, particularly over the right frontal pole and medial aspect of the right frontal lobe. Stripping of the meninges from the right frontal pole revealed gross atrophy of the gyri, simulating a microgyria. The cortical surface was pitted with many tiny sunken scars, giving a thimble-like appearance (Fig. 2). Sections of the cerebrum showed extensive necrosis of the cortex, affecting almost the whole of the right and most of the left frontal lobes, sparing the left supracallosal and orbital gyri. A large, elongated focus of softening, $3 \times 1 \times 1 \mathrm{~cm}$., was present in the right cingular area and was associated with several small, white, thread-like vessels $(1 \mathrm{~mm}$. in diameter) at the periphery. The temporal lobes, apart from the atrophic cortex on either side of the collateral fissure, appeared normal. The parietal and occipital cortex on both sides was extremely atrophic, being in places no more than

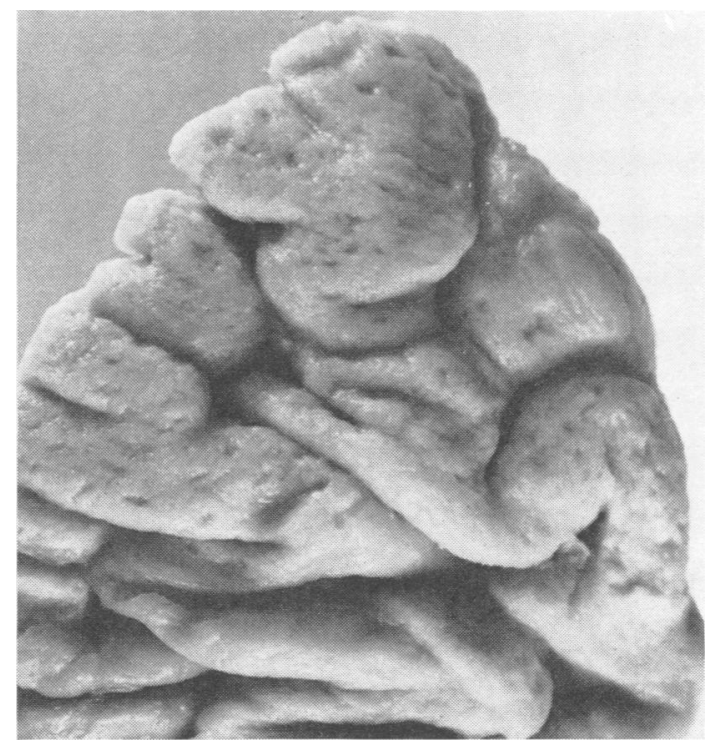

FIG. 2.-The right frontal lobe; numerous thimble-like cortical scarsgranular atrophy. About $\times 3.5$. 


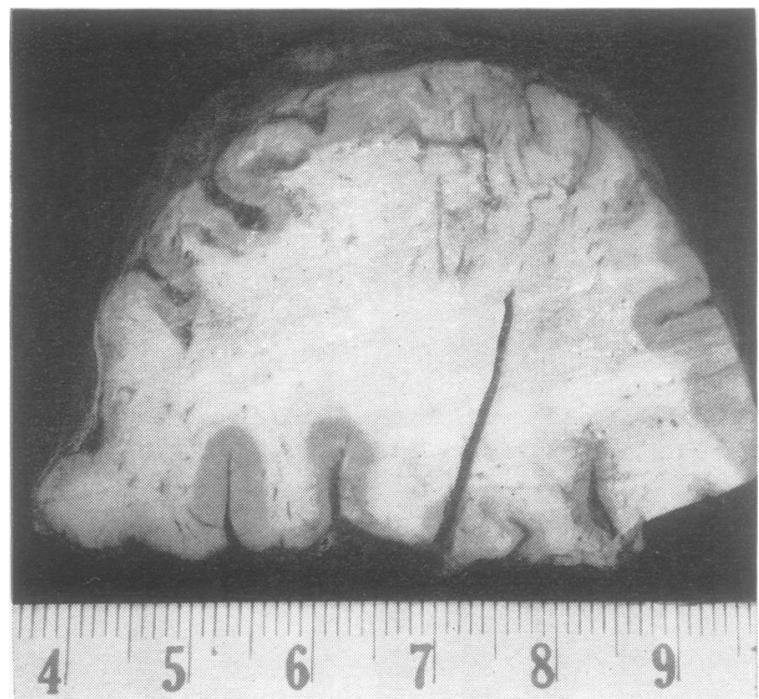

Fig. 3.-Right parietal cortex (coronal section) showing cortical and subcortical infarction, most marked laterally.

$1 \mathrm{~mm}$. thick. The convexity was, on the whole, more affected than the base (Fig. 3), but the atrophy was generalized, sparing only the lateral aspects of the temporal lobes. The internal carotid and main trunks of the anterior, middle, and posterior cerebral arteries were free of antemortem or organized thrombus. The cerebellum showed externally little obvious change apart from being small. Section showed diffuse loss of outline of the folia, more severe on the right side, resulting from necrosis. The cerebellar changes were less easily appreciable than those in the cerebrum. The basal ganglia, the midbrain, pons and medulla showed no gross abnormalities. There was a generalized dilatation of the ventricular system.

The heart weighed $360 \mathrm{~g}$. There was dilatation of the right side and concentric hypertrophy of the left ventricle. The myocardium was very tough and pale. The valves and coronary arteries appeared healthy.

The lungs showed bronchopneumonia in the left lower lobe and a small lung abscess in the base of the right lower lobe.

The kidneys were very granular and mottled (weights not recorded). The bladder showed chronic infection.

The spleen contained some old scars.

The endocrine glands, gastro-intestinal tract, and the liver showed no gross significant abnormalities.

Histology.-Frozen, paraffin, and celloidinembedded sections were stained with haematoxylin and eosin, Weigert's elastic stain and van Gieson, Nissl's stain for neurons, phospho- tungstic-acid-haematoxylin for glial fibres, Loyez's stain for myelin, and Gros-Bielschowsky's stain for axis cylinders.

Brain.-The maximal changes are in the right cingular, right parietal, and occipital cortical regions, and in the cerebellum. Changes are less marked in the temporal cortex and slight in the basal ganglia, midbrain, and pons.

The leptomeninges show great thickening by fibrosis, particularly in the right cingular and right parietal areas. There is an area of recent subarachnoid haemorrhage in the right cingular area. Extensive pigment depositionextracellular haematoidin and intracellular haemosiderin -is present in all severely affected areas, the pigment being concentrated perivascularly. There are some scattered particles of calcium in the fibrous occipital meninges. The meningeal changes are slight over the cerebellum compared with those of the cerebrum.

The blood vessels of the leptomeninges show more changes than those of the parenchyma, arteries being more conspicuously affected than veins. Some of the arteries are very tortuous, several loops being cut in one plane. Many vessels contain recanalized cellular plugs (Figs. 4 and 5). In some vessels concentric intimal proliferation is difficult to distinguish from an organized plug. One artery shows fairly recent organization of a plug, which contains many foam cells, the media of the vessel being infiltrated by lymphocytes and macrophages. ThE internal elastic layer is mostly well preserved, but ird some vessels there is poor staining and in others there
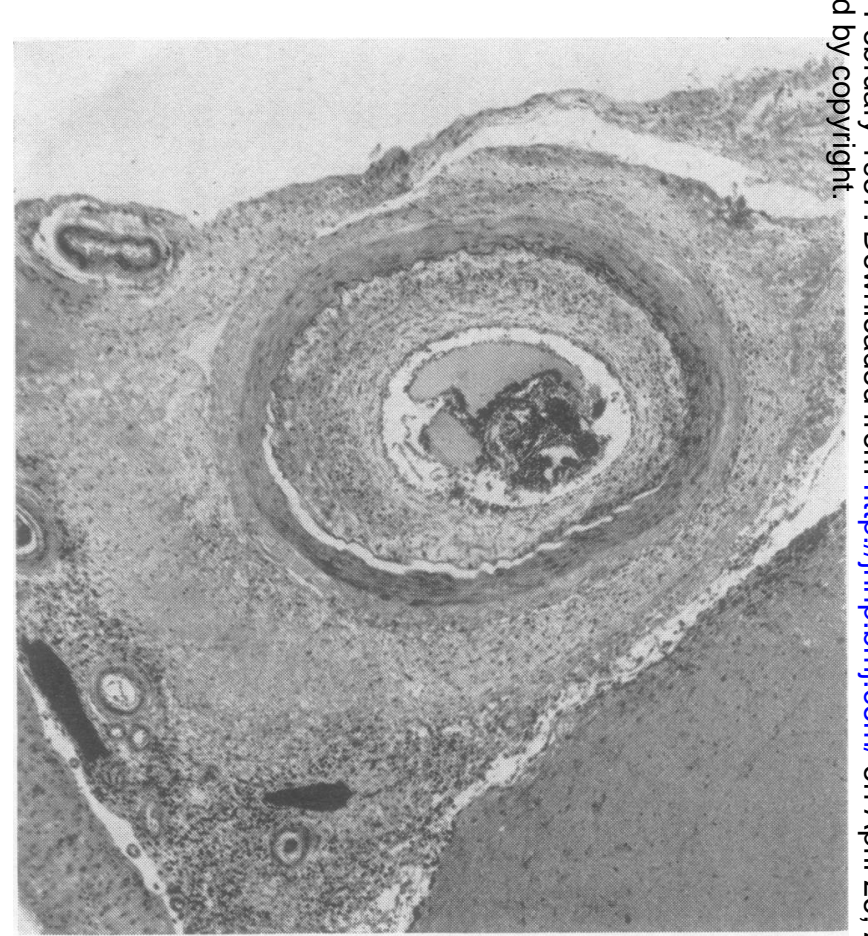

Fig. 4.-A thrombosed and recanalized meningeal artery. Note the cellularity of the tissue in the lumen. Haematoxylin and eosin $\times 40$. 
are gaps in this membrane; some of these vessels are plugged, others are not.

The changes in parenchymal blood vessels are confined to the arterioles in the white matter and in the basal ganglia. One vessel in the right retrocentral white matter shows recent fibrin thrombus in its lumen. Nearby, another vessel showed perivascular cuffing by macrophages, some containing haemosiderin. In the right caudate nucleus there is an arteriole plugged by what seems to be a recanalized thrombus, and others with eccentric intimal thickening and small perivascular haemorrhages. There is medial thickening and hyalinosis, and some intimal proliferation. An arteriole in the pons shows some eccentric hyaline material projecting into the lumen. A vessel in the choroid plexus is plugged and the lumen is partly obliterated. Cerebellar blood vessels show little conspicuous change. There is no evidence of atheroma in any of the sections examined.

The parenchyma shows changes corresponding in severity with the vascular distribution, the most severe changes being seen in the right cingulum, parietal, and occipital cortices. There are varying degrees of cortical infarction, ranging from complete liquefaction with replacement by compound granular corpuscles and gemästete astrocytes to oedematous areas with deposition of haematoidin and haemosiderin, and areas of scarring, the glial fibres lying largely vertical to the surface in the granular atrophic areas. Sometimes extensive superficial

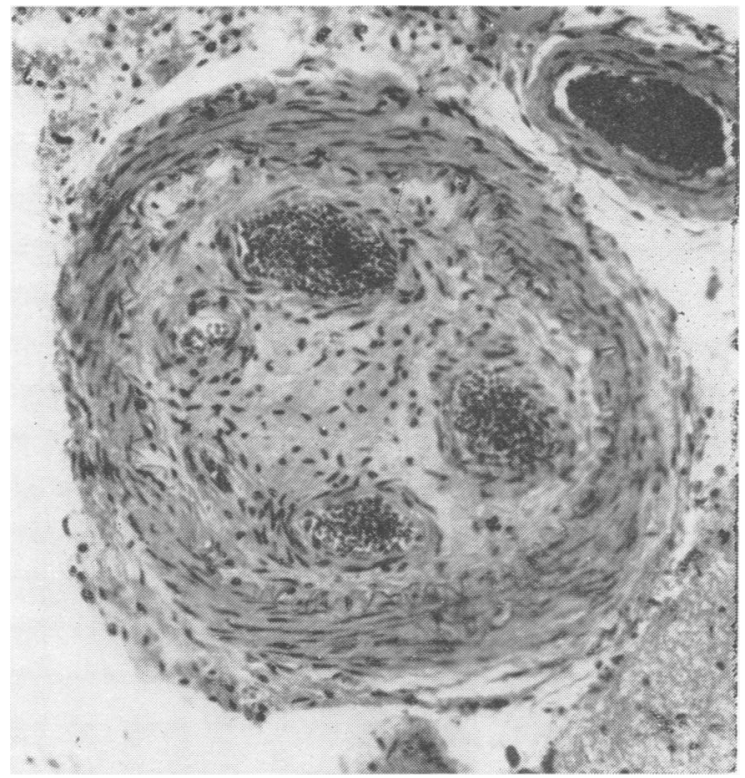

Fig. 5.-A thrombosed meningeal vessel with five new channels. Note the cellular Füllgewebe. Haematoxylin and eosin $\times 160$.

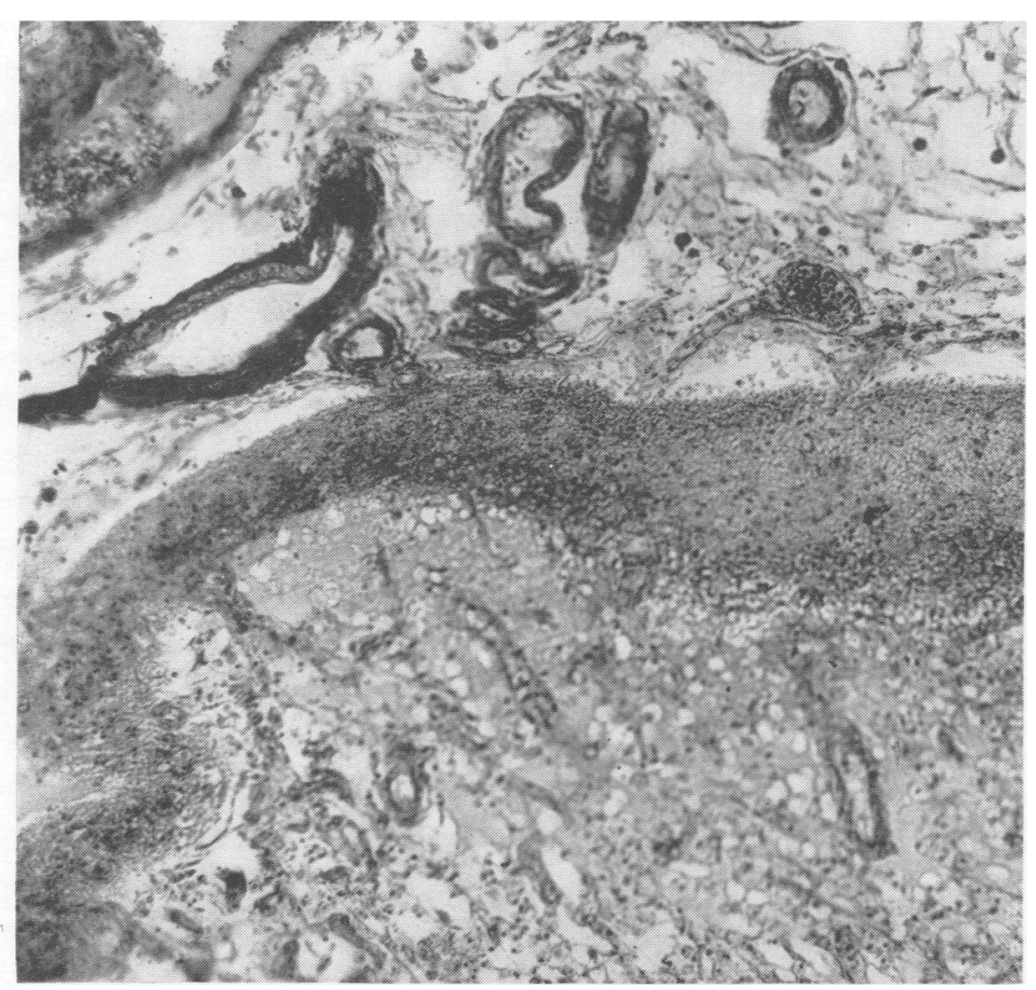

FIG. 6.-Parietal cortex with liquefaction necrosis deep to a border rim of gliosis. The adjacent vessels are meningeal. P.T.A.H. $\times 90$. gliosis forms a rim or border round the gyrus (Fig. 6). In less damaged areas most of the surviving neurons in the nucleus are swollen with loss of Nissl substance. The white matter shows oedema and early infarction, with compound granular corpuscles and gemästete astrocytes in some areas, notably in the right cingular and right basal ganglionic regions. Patchy demyelinization, often leaving a wide perivascular zone, is seen in the more severely affected areas. A patchy loss of axis cylinders with no special distribution is seen in various parts of the hemispheres.

The cerebellum shows changes in both hemispheres, the right being the more severely involved. There is widespread infarction of the molecular layer extending sometimes into the granular layer to involve the whole folium. Phosphotungstic - acid - haema toxylin staining shows that there is severe gliosis of the molecular layer (resembling the rim of gliosis in the cerebral cortex) with a comparatively 


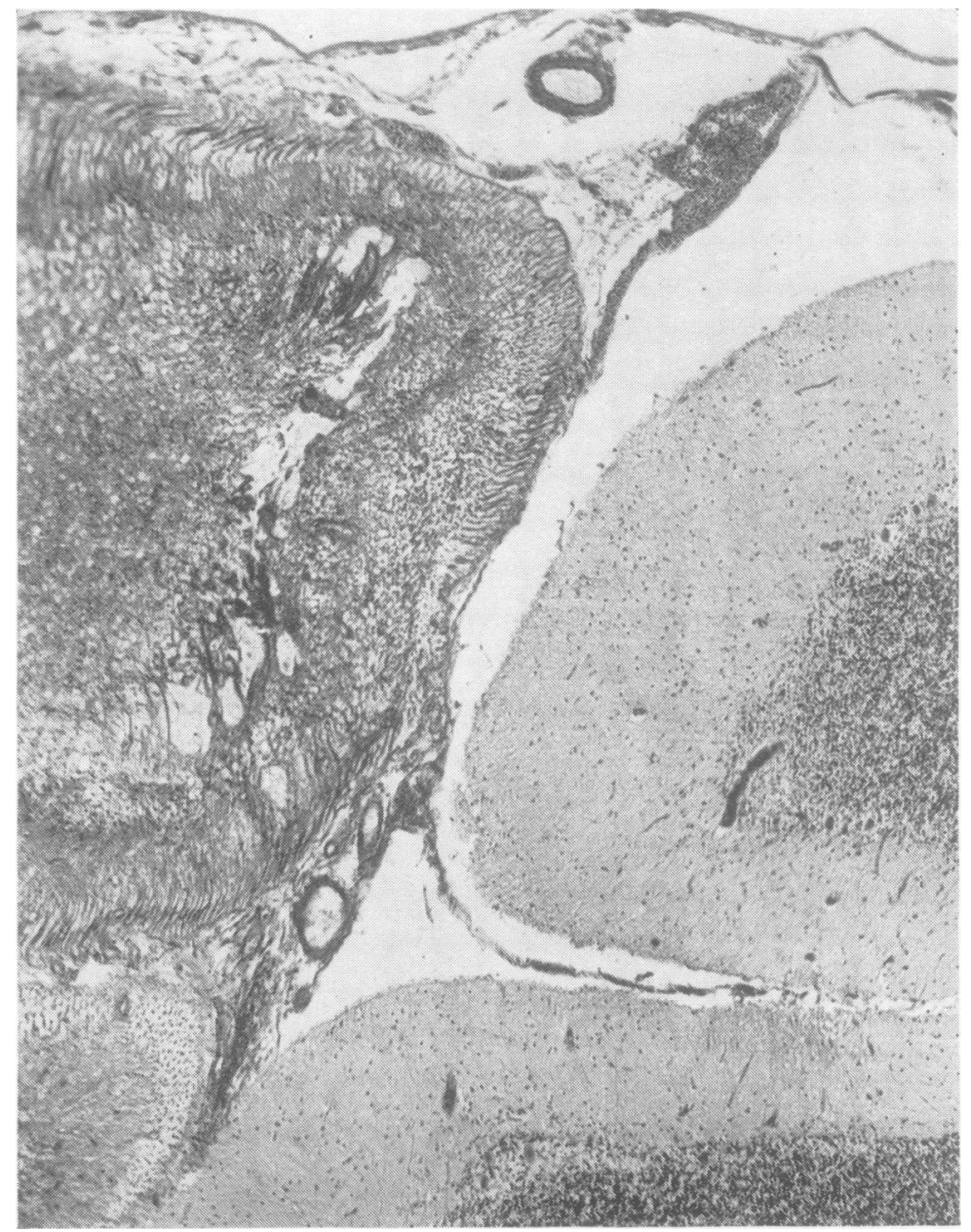

Fig. 7.-Cerebellum: a normal folium is seen on the right. The necrotic folium on the left shows thick fibres of gliosis running at right angles to the surface in the molecular layer. P.T.A.H. $\times 12$.

empty granular area in the centre of the folium, giving an almost cystic appearance (Fig. 7). Less severely affected areas show numerous small scars vertical to the surface. These are usually perivascular and sometimes extend into the granular layer, where there are collections of foam cells in the scar tissue (Fig. 8). These less severely damaged areas often show a marked outfall of Purkinje cells and of granular cells.

The optic nerve and blood vessels of the limbs were not available for examination.

Other Organs.-There are many scattered fibrous areas in the myocardium, some quite large. Some coronary arteries show eccentric intimal thickening. One large artery shows a well organized, recanalized plug. A section of heart valve shows no evidence of endocarditis.

Some glomeruli show thickening of capsules and of basement membranes; a fair number of peripheral glomeruli are fibrosed and partly hyalinized. There are occasional collections of neutrophils in the tubules, with congestion and interstitial fibrosis. Most of the arcuate and many interlobular arteries show concentric or eccentric intimal thickening of the endarteritis fibrosa type, and many arcuate vessels are obliterated by recanalized plugs (Fig. 9). No recent infarcts or thromboses are seen. The elastica interna of many of these vessels are split and there are some segmental gaps (Fig. 10); arterioles and capillaries show no obliterative or necrotizing changes.

Sections of spleen show healed infarction and recanalized plugging or proliferated intima in trabecular vessels. The suprarenal, tongue, testis, and portal tracts show eccentric intimal thickening and medial hyalinosis of arterioles, with occasional recanalized plugs.

Other organs show no significant abnormalities. 


\section{Discussion}

The onset of the disease was insidious and the course steadily progressive, without any acute and sudden incidents. The presenting symptom was dementia, with later development of visual failure (probably due to a chiasmal lesion), left hemiparesis, and marked left cerebellar ataxia. The symptomatology only involved the central nervous system. An intracranial tumour appeared to be a possibility although the hypertension, clearly of short duration, in a young adult was a puzzling feature. Tumour having been excluded, various forms of vascular disease had to be considered. There was no evidence of valvular heart disease or bacterial endocarditis as a source of possible emboli. Syphilis was excluded by serology. Either polyarteritis nodosa or malignant hypertension appeared to be the most probable diagnosis. The neurological picture was unlike that usually seen in polyarteritis nodosa, in which polyneuritis, convulsions, or acute cerebral vascular incidents are the commonest manifestations (Miller and Daley, 1946). The absence of hypertensive retinopathy and of

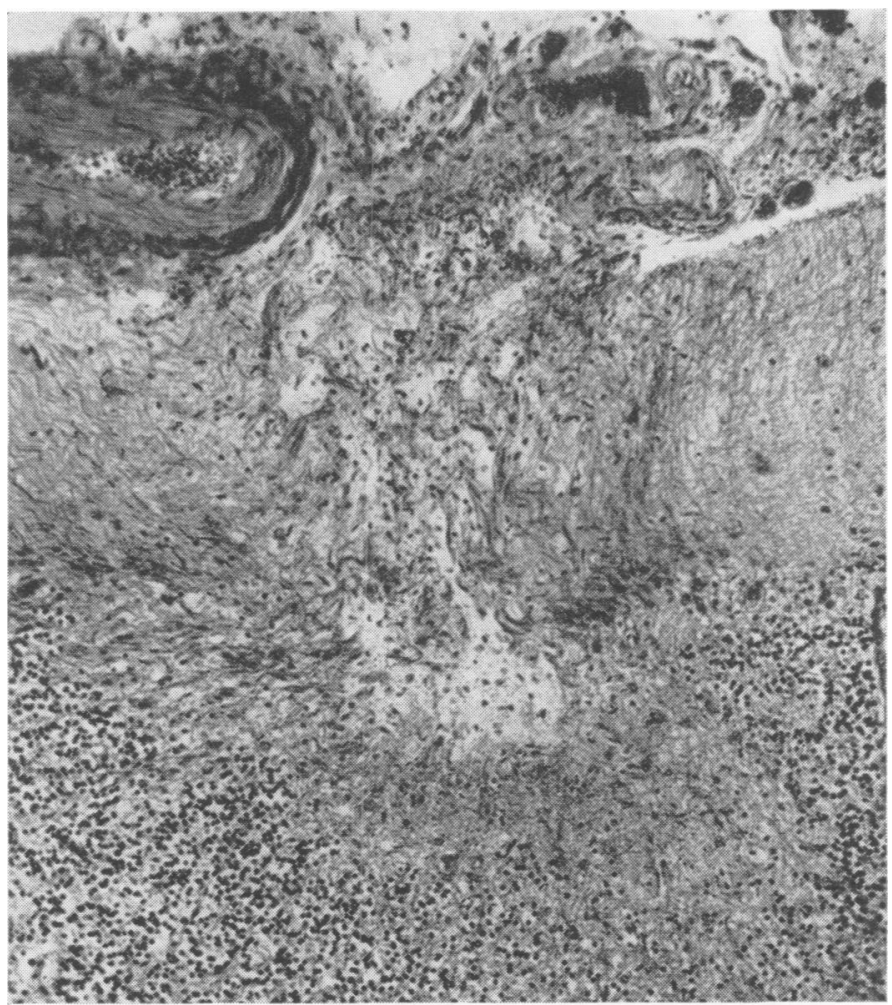

FIG. 8.-Cerebellum: a broader glial scar extending into the granular layer. The scarred area contains many foam cells. There is an almost total loss of Purkinje cells. P.T.A.H. $\times 60$.

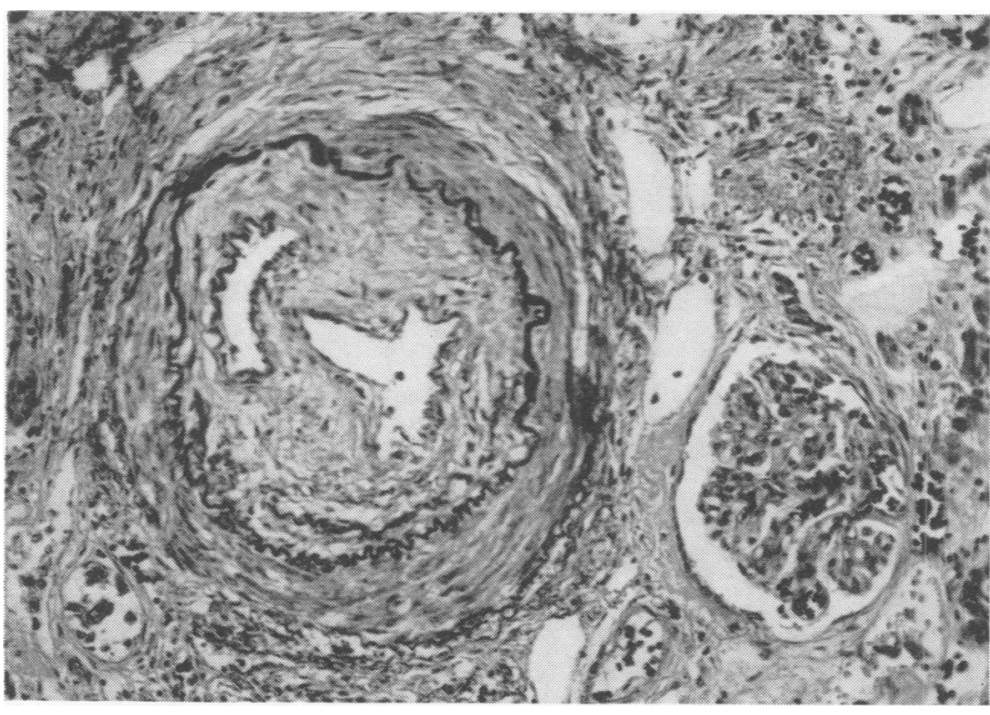

Fig. 9.-Recanalized artery in kidney. The elastic layer is split and stained poorly. Weigart-van Gieson $\times 160$. marked renal failure seemed strongly against malignant hypertension. Thromboangeitis obliterans was not considered clinically, but in retrospect many of the features are suggestive of cases of this condition with cerebral involvement described in the literature (Lindenberg and Spatz, 1939; Antoni, 1941; Minkowski, 1947-48; Llavero, 1948); the onset with dementia, progressive course, signs of focal multiple lesions, tendency to cerebellar involvement, and hypertension.

Pathologically, the gross appearance in the brain was that of atrophy, due to widespread cortical necrosis, more severe on the right side. The haemosiderin staining and thickened, 


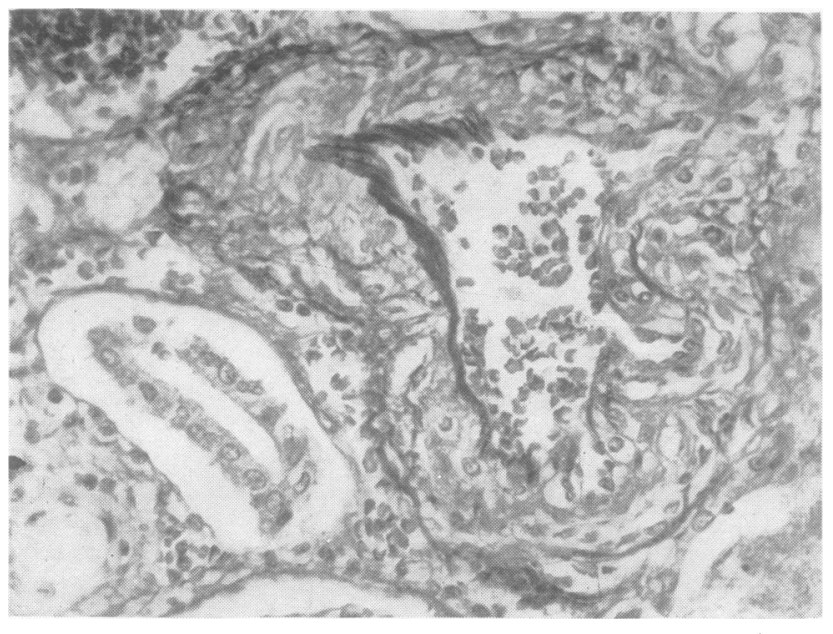

FIG. 10.- Renal artery showing medial hypertrophy segmental elastic gaps. Weigart-van Gieson $\times 160$.

white, wormlike vessels near the large focal lesion of the right cingulum suggested a vascular lesion. The largely cortical involvement with relative sparing of white matter, and the absence of antemortem thrombi in the large vessels-internal carotid and major cerebral arteries-indicated that small peripheral branches were affected. The curious pitted, thimble-like appearance of the frontal cortex is due to focal cortical scarring at right angles to the surface and is known to continental writers as "granular atrophy". Lindenberg and Spatz (1939) stress this appearance as characteristic of cerebral thromboangeitis obliterans, though Morel (1946) considers it can arise from other causes of intermittent insufficiency of the cerebral circulation. The small cerebellar scars are probably analogous to granular atrophy in the cerebrum.

Histologically, the picture was of largely cortical necrosis with relative sparing of central white matter, basal ganglia, and brain-stem. The vascular lesions seemed wholly to account for the necrosis. The vessels chiefly involved were pial arteries, with an occasional thrombosed arteriole or inflamed arteriole in the white matter. The scarcity of arteriolar and capillary lesions excludes Goodman's (1949) " recurrent hypertensive cerebral thrombosis" in which arteriolar lesions predominate. Atherosclerosis was not present histologically and syphilis was excluded serologically. No endocardial lesion, liable to produce embolism, was found (though myocardial fibrosis and some coronary sclerosis were present). In considering malignant hypertension, polyarteritis nodosa, and thromboangeitis obliterans, it is necessary to take the changes in the brain together with those in other organs, notably the kidneys. The latter showed severe contraction, with glomerular fibrosis, and obliterative endarteritis, affecting arcuates and interlobulars. Recanalization was conspicuous, as it was in the brain. Necrotizing arteriolitis was not seen in the kidneys or in any other organ. This would seem to be an important point against malignant hypertension running such an acute course, though changes resembling endarteritis fibrosa were present. Gaps in the elastica interna were present in vessels of both brain and kidney, and suggested the possibility of healed polyarteritis nodosa (Pagel, 1951); no pictures of active polyarteritis nodosa (acute inflammatory infiltration with fibrinoid necrosis) were seen. Secondary thombosis occurs in this condition when accompanied by hypertension (Davson, Ball, and Platt, 1948) and in primary malignant hypertension, though Heptinstall (1953) does not describe thrombosis as a characteristic finding in the latter condition. Thromboangeitis obliterans in the healed phase would show little to distinguish it from other thrombotic lesions of arteries, apart from its tendency to involve the arteries of the limbs, though this is not invariable. Unfortunately material from the limbs was not available, as this possibility had not been considered at the time of necropsy. The diagnostic stress laid by Jäger (1932) on the connective tissue filling the lumen of the thrombosed artery in thromboangeitis obliterans-the Füllgewebe-is probably too great; similar pictures may be found in hypertension due to any cause. Nevertheless, the gross and histological features of the brain accord quite closely with some cases described of thromboangeitis obliterans involving the brain, notably the granular atrophy. It is noteworthy that Jäger's Case 2 showed lesions of acute polyarteritis nodosa in the liver, kidneys, and brain, as well as widespread lesions of thromboangeitis obliterans. As thromboangeitis obliterans is considered by many (Llavero, 1948) to originate in hypersensitivity, overlapping of histological lesions in the two diseases may well be possible. It seems likely that the disease of our patient was of this nature, the hypertension being secondary to the renal ischaemia following the obliterative arterial disease, rather than primary. There is no clue to any possible sensitizing process, and the family history was negative both for allergy and hypertension.

On the whole we feel that the weight of both clinical and pathological evidence in this case favours a diagnosis of thromboangeitis obliterans but there can be no sharp pathological distinction between this disease and polyarteritis nodosa in their later stages. 


\section{Summary}

A young man, aged 19 years, died after an illness lasting 13 months, in which progressive dementia, optic atrophy, left cerebellar ataxia, left hemiparesis, and hypertension were the outstanding features.

Pathologically, there was diffuse cortical necrosis with granular atrophy of the brain, nephrosclerosis, and widespread obliterative lesions of medium-sized arteries in the involved organs.

The vascular lesions suggested either polyarteritis nodosa or thromboangeitis obliterans in their later stages.

The differential diagnosis is discussed.

\section{REFERENCES}

Antoni, N. (1941). Acta med. scand., 108, 502.

Davson, J., Ball, J., and Platt, R. (1948). Quart. J. Med., n.s., 17,

Goodman. L. (1949). Arch. Neurol. Psychiat. (Chicago), 62, 445.

Heptinstall, R. H. (1953). J. Path. Bact., 65, 423.

Jäger, E. (1932). Virchows Arch. path. Anat., 284, 527.

Jäger, E. (1932). Virchows Arch. path. Anat., 284, 527.

Llavero, F. (1948). Thromboendangitis obliterans des Gehirns. Benno Schwabe, Basel.

Miller, H. G., and Daley, R. (1946). Quart. J. Med., n.s., 15, 255.

Minkowski, M. (1947-48). Confinia Neurologica, 8, 138

Morel, F. (1946). Schweiz. Arch. Neurol. Psychiat., 57, 350.

Pagel, W. (1951). J. clin. Path., 4, 137.

\section{THE NOVEMBER (1956) ISSUE}

The November (1956) issue contains the following papers:-

Pearly Tumours in Relation to the Central Nervous System. John S. Tytus and Joe Pennybacker.

Physiological Tremor. John Marshall and Geoffrey Walsh.

Subacute Myoclonic Spinal Neuronitis. A. M. G. Campbell and Hugh Garland.

Electrical Signs in the Diagnosis of Carpal Tunnel and Related Syndromes. John A. Simpson.

Ischaemic and Postischaemic Paraesthesiae in Polyneuritis. E. W. Poole.

The Pupillary Changes in the Holmes-Adie Syndrome. G. F. M. Russell.

Sensory Deficits in Visual Agnosia. G. Ettlinger.

Inappropriate Affect. Arthur Harris and Maryse Metcalfe.

Book Reviews.

Index.

A number of copies are still available and may be obtained from the Publishing Manager, British Medical Association, Tavistock Square, W.C.1, price 12s. $6 \mathrm{~d}$. 